\title{
Inflammatory fibroid polyp: A case report with review of the literature
}

\author{
(1) Bita Jafarzadeh ${ }^{1}$, (1) Masoud Sadeghi ${ }^{2,3}$, (1) Mazaher Ramezani ${ }^{1}$ \\ ${ }^{1}$ Kermanshah University of Medical Sciences, Imam Reza Hospital, Molecular Pathology Research Center, Kermanshah, Iran \\ ${ }^{2}$ Kermanshah University of Medical Sciences, Medical Biology Research Center, Kermanshah, Iran \\ ${ }^{3}$ Kermanshah University of Medical Sciences, Students Research Committee, Kermanshah, Iran
}

\section{Date submitted: \\ 19.07.2019}

Date accepted:

18.09.2019

Online publication date:

15.03.2020

\section{Corresponding Author:}

Mazaher Ramezani MD, Kermanshah University of Medical Sciences, Imam Reza Hospital, Molecular Pathology Research Center, Kermanshah, Iran mazaher_ramezani@yahoo.com

ORCID:

orcid.org/0000-0003-1897-7410

Keywords: Inflammatory fibroid polyp, stomach, Vanek's polyp, case report

\begin{abstract}
Inflammatory fibroid polyps (IFPs) are rare benign mesenchymal neoplasms that occur anywhere in the gastrointestinal tract but most commonly in the stomach and small intestine. Here, in this study, we reported a case of IFP in gastric antrum in an Iranian woman in Western Iran. The report included a 59-year-old woman with a chief complaint of dyspepsia. The upper endoscopy revealed "gastric antrum polyp" and the previous pathology report was "adenomatous polyp (tubular adenoma) with low-grade dysplasia". In conclusion, IFP is a benign submucosal lesion occurring more frequently in $6^{\text {th }}$ or $7^{\text {th }}$ decade of life, which is rarely associated with adenocarcinoma or adenoma and is been treated by local excision.
\end{abstract}

\section{Introduction}

Inflammatory fibroid polyp (IFP) or Vanek's polyp is a rare polyp, the etiology of which is not fully understood (1). IFP can arise everywhere in the gastrointestinal tract but is described more frequently in the gastric antrum (2). Its incidence rate is extremely low, from $0.1 \%$ to $2 \%$ (3). IFPs may be seen at any age but mainly in the fifties to sixties (2). Gastric IFPs are usually asymptomatic or present with nonspecific symptoms (4). The endoscopic investigation only shows nonspecific submucosal polyp (5). Macroscopically, the tumor size is from 0.2 to $4.2 \mathrm{~cm}$ with a mean size of $1.7 \mathrm{~cm}$ (6). Microscopically, the lesions are located in submucosa and composed of spindle and inflammatory cells. Spindle cells are whorled around vasculature with onion skin pattern. Inflammation contains many eosinophils. The background is fibromyxoid and atypia is none with rare mitoses if any $(6,7)$. The spindle cells are immunoreactive for the cluster of designation (CD) 34 and negative for CD117 and also smooth muscle actin (SMA) staining is variable $(6,8)$. Recent data show that the spindle cells express platelet-derived growth factor receptor alpha (PDGFRA). This activating mutation is seen in the majority of IFP cases. Therefore, IFP represents true benign mesenchymal tumors of the gastrointestinal tract (9). Although it is rarely associated with adenocarcinoma or adenoma, local excision is usually curative (10). This study reported a case of IFP in gastric antrum in an Iranian woman in Western Iran.

\section{Presentation of Case}

A 59-year-old woman was admitted to the department of gastroenterology on July $26^{\text {th }}, 2018$ with a chief complaint of dyspepsia lasting for 2 years. There was no significant past 
medical history or drug history. The physical examination was unremarkable. The lab data such as complete blood count, thyroid function tests, biochemistry, and coagulative function analysis were normal. The patient underwent upper gastrointestinal endoscopy on $28^{\text {th }}$ August, which was reported as "antral polyp" (M: $1.5 \times 1 \times 0.5 \mathrm{~cm}$ ) and the pathology was reported as "Adenomatous Polyp (tubular adenoma) with low-grade dysplasia". The second endoscopy was done on 6th October, reporting an "antral polyp" (M: $1 \times 0.5 \times 0.5 \mathrm{~cm})$ and the pathology was reported as IFP. Grossly the specimen consisted of multiple grayish-tan fragments measuring $1 \times 0.5 \times 0.5 \mathrm{~cm}$. Microscopic examination on the $21^{\text {th }}$ showed "Submucosal lesion of loose edematous stroma that composed of spindle stromal cells with thin-walled blood vessels and "onion skin" arrangement around vessels and Inflammatory infiltration rich in eosinophils, with no atypia and mitotic activity", which was compatible with the diagnosis of IFP (Figure 1). The immunohistochemistry (IHC) staining of CD34 was positive, whereas CD117, cytokeratin, and SMA were negative in favor of the diagnosis (Figure 2). The treatment process was thoroughly explained to the patient, and written informed consent was obtained prior to the onset of treatment. Moreover, the patient was informed about reporting the case and consented to the publication of photographs and radiographs.

\section{Discussion}

IFP is a rare, usually solitary, sessile or pedunculated submucosal polyp, which was proposed by Helwig and Ranier as "inflammatory fibroid polyp" in 1953 (8). It was initially reported by Vanek (7) in 1949, as it is clinically called Vanek's tumor, and histologically was called "gastric submucosal granuloma with eosinophilic infiltration" (11). We reported a case of IPF of gastric antrum in a 59-year-old woman with two-year dyspepsia without a significant past medical history. This tumor is more prevalent in

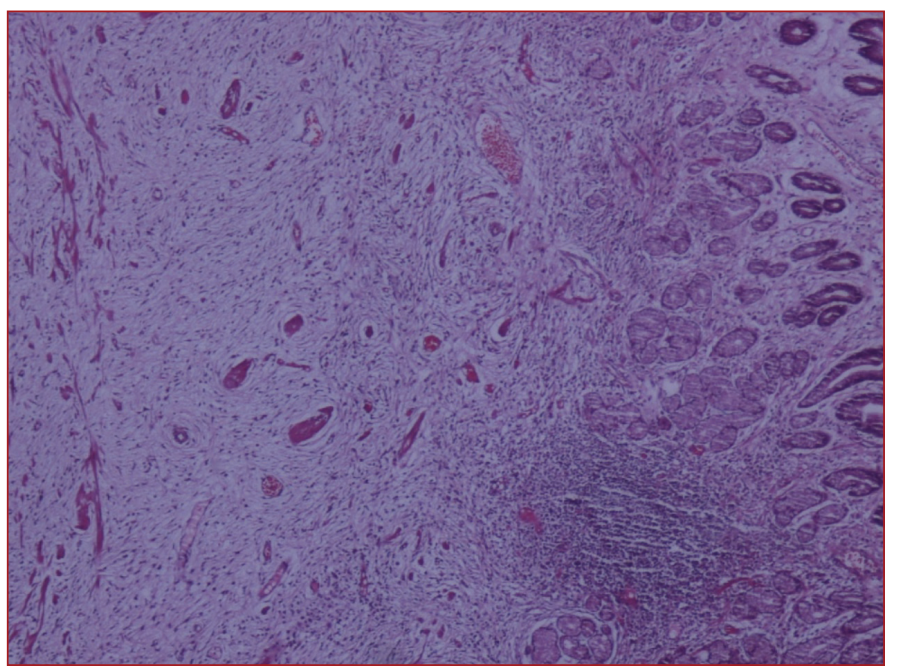

Figure 1. Spindle cells around vasculature and inflammatory cells containing eosinophils. Hematoxylin-Eosin staining (x40 magnification) middle-aged females, with the mean age in 60 s $(2,12)$. It occurs anywhere in gastrointestinal tract but most commonly in stomach, and it constitutes $2 \%$ of all gastric polyps. It usually arises in antrum $(70 \%)$ adjacent to pyloric sphincter, followed by the small and large bowel (25\%), gallbladder (1\%), esophagus $(1 \%)$, duodenum (1\%), anal canal (1\%), and appendix (2). Although exact pathogenesis is unknown, one study suggested a familial basis, referred to as Devon polyposis (13). Clinical symptoms and endoscopic appearances are usually nonspecific (14). IFPs are submucosal with an average diameter of $1.7 \mathrm{~cm}$, most lesions are smaller than $3 \mathrm{~cm}$. Microscopically, it is a submucosal lesion with an abrupt demarcation of the muscularis propria and is characterized by a vascular and fibroblastic proliferation (often in a whorl-like arrangement around blood vessels) and a polymorphic inflammatory response, dominated by eosinophils in an edematous background $(6,7,14)$. The differential diagnosis includes eosinophilic gastroenteritis, gastrointestinal stromal tumor, inflammatory pseudotumor, hemangioendothelioma, and hemangiopericytoma $(2,14)$. In IHC staining, stromal cells are positive for PDGFR, CD34, vimentin, fascin, CD35, cyclin D1, and calponin and negative for CD117 (14). Although it is rarely associated with adenocarcinoma or adenoma, local excision is usually curative, and no endoscopic surveillance is required after the histological diagnosis is confirmed. However, Mori et al. (10) emphasized that association between IFP and adenoma or adenocarcinoma was possible and they showed this in 6 cases. Mucientes et al. (15) stated that only 7 cases were on the literature for association of IFP and gastric carcinoma and they reported another case of early gastric carcinoma underlying IFP. Association between IFP and adenoma or carcinoma is limited to case reports. Whether this association is an incidental finding or not needs to be clarified by further studies.

IFP occurs more frequently in middle-aged adults and is more common in the gastric antrum. The incidence is extremely

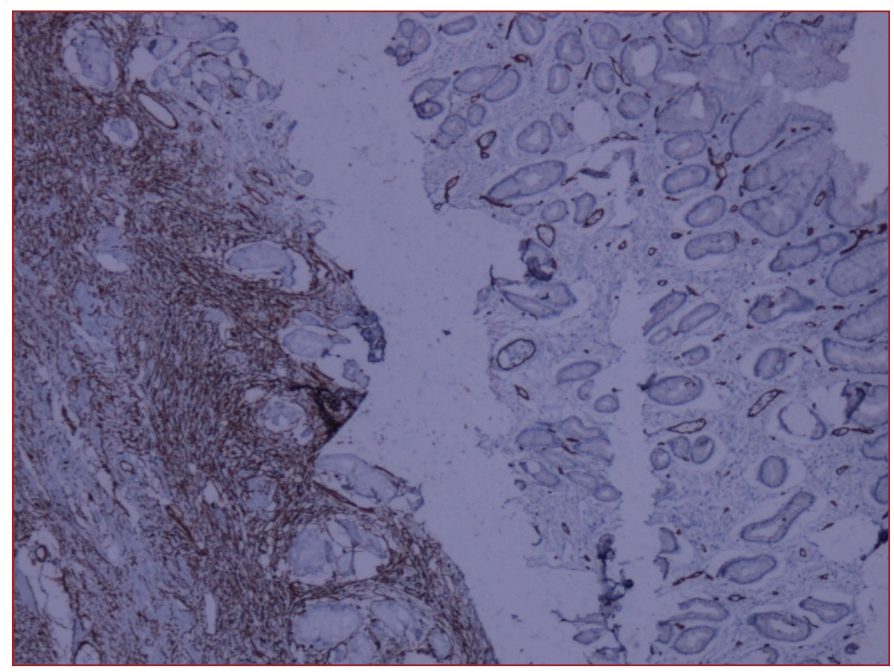

Figure 2. CD34 positive cells. Immunohistochemistry staining ( $x 40$ magnification) 
low: from $0.1 \%$ to $2 \%$. Clinical manifestations are heterogeneous and endoscopic examination reveals only the presence of a submucosal lesion. Clinical, endoscopic, and histopathologic correlation would lead to the diagnosis of IFP. The IHC staining is useful in confirmation and ruling out of the differential diagnosis. IFP is a benign lesion and is been treated by local excision.

\section{Ethics}

Informed Consent: Consent form was filled out by all participants.

Peer-review: Externally peer-reviewed.

\section{Authorship Contributions}

Concept: M.R., B.J., Design: M.R., B.J., Data Collection or Processing: M.R., B.J., Analysis or Interpretation: M.R., M.S., Literature Search: B.J., Writing: M.R., B.J., M.S.

Conflict of Interest: No conflict of interest was declared by the authors.

Financial Disclosure: The authors would like to thank the Clinical Research Development Center of Imam Reza Hospital for Consulting Services.

\section{References}

1. Rizzo G, Fancellu A, Porcu A. Inflammatory fibroid polyp (Vanek's tumor) of the gastric antrum: is treatment always mandatory? Ann Ital Chir. 2016:87.

2. Pinto-Pais T, Fernandes S, Proença L, et al. A Large Gastric Inflammatory Fibroid Polyp. GE Port J Gastroenterol. 2015;22:61-64.

3. Rossi P, Montuori M, Balassone V, Ricciardi E, Anemona L, Manzelli A, Petrella G. Inflammatory fibroid polyp. A case report and review of the literature. Ann Ital Chir. 2012;83:347-351.

4. Godey SK, Diggory RT. Inflammatory fibroid polyp of the oesophagus. World J Surg Oncol. 2005;3:30.
5. Matsushita M, Hajiro K, Okazaki K, Takakuwa H. Gastric inflammatory fibroid polyps: endoscopic ultrasonographic analysis in comparison with the histology. Gastrointest Endosc. 1997;46:53-57.

6. Liu TC, Lin MT, Montgomery EA, Singhi AD. Inflammatory fibroid polyps of the gastrointestinal tract: spectrum of clinical, morphologic, and immunohistochemistry features. Am J Surg Pathol. 2013;37:586-592.

7. Vanek J. Gastric submucosal granuloma with eosinophilic infiltration. Am J Pathol. 1949;25:397-411.

8. Helwig EB, Ranier A. Inflammatory fibroid polyps of the stomach. Surg Gynecol Obstet. 1953;96:335-367.

9. Schildhaus HU, Merkelbach-Bruse S, Binot E, Büttner R, Wardelmann E. Inflammatory fibroid polyp: from Vanek's "submucosal granuloma" to the concept of submucosal mesenchymal neoplasia. Pathologe. 2010;31:109-114.

10. Mori M, Tamura S, Enjoji M, Sugimachi K. Concomitant presence of inflammatory fibroid polyp and carcinoma or adenoma in the stomach. Arch Pathol Lab Med. 1988; 112:829-832.

11. Banik S, Temple JG. Inflammatory fibroid polyp of the stomach. Br J Clin Pract. 1979;33:178-181.

12. Stolte M, Finkenzeller G. Inflammatory fibroid polyp of the stomach. Endoscopy. 1990;22:203-207.

13. Allibone RO, Nanson JK, Anthony PP. Multiple and recurrent inflammatory fibroid polyps in a Devon family ("Devon polyposis syndrome”): an update. Gut. 1992;33:10041005.

14. Miettinen $M$, Sobin LH, Sarlomo-Rikala M. Immunohistochemical spectrum of GISTs at different sites and their differential diagnosis with a reference to CD117 (KIT). Mod Pathol. 2000;131134-1142.

15. Mucientes $P$, Mucientes F, Klaassen R. Inflammatory fibroid polyp associated with early gastric carcinoma: a case report. Ann Diagn Pathol. 2012;16:148-151. 